\title{
Modern aspects of testing azole antifungals
}

\author{
I. HALLER \\ Dr rer. nat. \\ Institute for Chemotherapy, Bayer AG, D-5600 Wuppertal, Germany
}

\begin{abstract}
Summary
Difficulties in the in vitro testing of azole antimycotics are described. The value of minimal inhibitory concentration results are discussed and contrasted to those obtained with polyene antimycotics.
\end{abstract}

\section{Introduction}

Minimal inhibitory concentration (MIC) serves as the main parameter to define the antimycotic efficacy of substances or to determine the susceptibility of fungal strains. Even if refined in vitro test methods were available they would not replace the use of the MICs, since clinical practice permits only rapid and simple methods of determination. However, the MIC is an artificial parameter, which does not satisfactorily reflect the physiological conditions prevailing during treatment of mycoses.

It has been known for many years that the in vitro inhibitory effect of imidazole antimycotics depends to a high degree on the test conditions chosen. Nutrient medium, size of inoculum, incubation period and reading method are factors of particular importance in this respect. Differences in the test conditions applied have often induced controversies about the efficacy of the azole antimycotics. The question as to what degree the in vitro effect of the azoles correlates, if at all, with the therapeutic action in vivo has, therefore, been examined.

\section{Methods and results}

\section{Determination of MIC in the broth dilution test}

The MIC is defined as the lowest concentration of active substance inhibiting the reproduction of the test organisms. Whether there has been growth or not is, however, usually ascertained with the naked eye. If cocci and rod-shaped bacteria are used a clearly visible clouding of the culture is produced by approximately $10^{7}$ cells $/ \mathrm{ml}$ or more. The budding cells of yeasts, however, sink to the bottom of the test tubes and $10^{6}$ cells are already discernible as a very thin whitish deposit. With an inoculum of $10^{5}$ yeast cells $/ \mathrm{ml}$ already 2 or 3 divisions of the cellular population-depending on the test volume-may suffice to make fungal growth visible. If, in addition, as is usual with the azoles, all transitory stages between total inhibition of growth and completely uninhibited growth occur, a quantitative assessment of the inhibitory effect will be difficult.

The MICs of clotrimazole and nystatin against several species of Candida (Table 1) and Torulopsis were determined under different test conditions.

TABLE 1. Minimal inhibitory concentration (MIC) ( $\mathrm{mg} / \mathrm{l})$ of clotrimazole and nystatin against Candida albicans by broth dilution testing

\begin{tabular}{|c|c|c|c|c|}
\hline \multirow[b]{3}{*}{$\begin{array}{l}\text { Inoculum } \\
10^{3} \text { cells } / \mathrm{ml}\end{array}$} & \multicolumn{4}{|c|}{ Incubation period } \\
\hline & \multicolumn{2}{|c|}{ Clotrimazole } & \multicolumn{2}{|c|}{ Nystatin } \\
\hline & $\begin{array}{c}2 \text { days } \\
0.25 \\
(0.062)\end{array}$ & $\begin{array}{c}3 \text { days } \\
1 \\
(0.5)\end{array}$ & $\begin{array}{c}2 \text { days } \\
0.5 \\
(0.25)\end{array}$ & $\begin{array}{c}3 \text { days } \\
0 \cdot 5 \\
(0 \cdot 5)\end{array}$ \\
\hline $10^{5}$ cells $/ \mathrm{ml}$ & $\begin{array}{r}32 \\
(4)\end{array}$ & $\begin{array}{r}32 \\
(4)\end{array}$ & $\begin{array}{l}0.5 \\
(0.5)\end{array}$ & $\begin{array}{l}0.5 \\
(0.5)\end{array}$ \\
\hline
\end{tabular}

The inoculum was $10^{3}$ and $10^{5}$ cells per ml respectively, and the incubation period 2 and 3 days. All 3 parameters (size of inoculum, incubation period and reading method) affect the MIC of clotrimazole so that considerable variations of the MIC values may result: in this case values ranged from 0.062 to $32 \mathrm{mg} / \mathrm{l}$. A similar dependence on the individual test conditions was observed for miconazole. The in vitro effect of nystatin and amphotericin B, on the other hand, proved to be largely independent of the varying test conditions.

The broth dilution tests were also quantitatively evaluated by means of a spectrophotometer. As was demonstrated by preliminary tests, the extinction of yeast suspensions could be taken as a measure of the cell count contained in it. Only when the solutions were very turbid did the measurement of absorption yield lower values than the cell count by means of the counting chamber. In such cases dilutions were made and the values extrapolated.

The inhibitory effect of nystatin against Candida albicans ended rather abruptly as soon as the concentration was below the minimum inhibitory level. Clotrimazole, however, considerably reduced fungal growth even at concentrations far below the MIC. 
Although the MIC of clotrimazole against the C. albicans strain used was $32 \mathrm{mg} / \mathrm{l}$, fungal growth was reduced by as much as $80 \%$ at an active substance concentration of only $0.5 \mathrm{mg} / \mathrm{l}$. In many experiments with different species of yeasts similar partial inhibitory effects of all azole derivatives tested have been observed.

\section{Determination of MIC in solid nutrient medium}

The same gradual type of action of the azoles can be observed on solid nutrient media. In testing clotrimazole, the effect of the inoculum is striking and the gradually declining inhibitory action of the drug is clearly visible. Although fungal growth has occurred at very low concentrations of the active substance the cell count represents only a small fraction of growth control. Nystatin presents a completely different picture: the inoculum has had no effect and there is an abrupt transition from total inhibition of growth to a totally uninhibited growth of the fungus.

\section{Discussion}

The different molecular mechanisms of action of the 2 classes of substance are directly linked to their therapeutic effect. The cytoplasmic membrane of fungi contains sterols which have a considerable influence on the physical state and permeability of the membrane. On contact with the cell membrane, polyene molecules are very rapidly bound to these sterols. When a certain concentration of the active substance is exceeded, disorders occur in the structure of the membrane which cause changes in its permeability and thus lead to damage of the fungal cell. The azole antimycotics inhibit the biosynthesis of the sterols, probably at a demethylation stage. The result is a gradual depletion of sterols in the cytoplasmic membrane, as soon as the existent pools are exhausted. The period between addition of azoles and onset of action in vitro is 2 to $4 \mathrm{hr}$. A partial inhibition of sterol synthesis is possible at low concentrations of active substance. This combination of factors explains the gradual type of action which is characteristic of the azole antimycotics.

Animal experiments and clinical studies have repeatedly shown under in vivo conditions that in association with the host defence mechanisms a clear reduction of cell growth suffices either to prevent the spread of a fungus infection or to eliminate it. In other words, a mere reduction of fungal growth can produce therapeutic effects. This is in contradiction to the classical concept that the MIC of an active substance must be attained at the site of infection.

An example is given to demonstrate how little an isolated observation of MIC values is helpful for the assessment of the therapeutic efficacy of azole antimycotics. BAY k 5031 (an azole tested in connection with a screening programme) exhibited only a weak inhibitory effect in vitro against a strain of $C$. albicans which had also been used for the intravenous infection of mice in animal experiments. The MIC was $16-32 \mathrm{mg} / \mathrm{l}$ as compared with $0.062-0.5 \mathrm{mg} / \mathrm{l}$ for clotrimazole. Nevertheless, BAY k 5031 proved to be superior to clotrimazole with regard to its therapeutic efficacy in experimentally induced candidiasis of mice (therapeutic active dosage $12.5-25.0 \mathrm{mg} / \mathrm{kg}$ of BAY k 5031, and $50.0 \mathrm{mg} / \mathrm{kg}$ of clotrimazole). Experiments on the pharmacokinetics of the 2 preparations provided no satisfactory explanation 0 for this discrepancy. However, the partial inhibitory effects of BAY k 5031 in vitro were particularlyo pronounced and it was considered, therefore, that $\frac{1}{2} \frac{\vec{D}}{\mathbb{D}}$ the good therapeutic effect was due to the slowingo 3 down of fungal growth at subinhibitory concentrations.

Azole antimycotics are able considerably to reduce fungal growth at concentrations far below the MIC and, under physiological conditions, subinhibitory concentrations of the azoles can produce a good therapeutic effect whereas polyene antimycotics exhibit a different type of action: their MIC values can be better used to describe the inhibitory effect.

These results take on a particular significance in view of the endeavours to establish uniform test specifications in mycology. There are justifiable doubts whether test conditions equally appropriate for all antimycotics are feasible at all. As far as azoles suitable for systemic application are concerned, therapeutic efficacy is assessable only if, owing to favourable test conditions, the partial inhibitory effects described are recorded. 